\title{
PROBING THE INTERMEDIATE-AGE GLOBULAR CLUSTERS IN NGC 5128 FROM ULTRAVIOLET OBSERVATIONS
}

\author{
Soo-Chang Rey ${ }^{1}$, Sangmo T. Sohn ${ }^{2,3}$, Michael A. Beasley ${ }^{4}$, Young-Wook LeE ${ }^{2}$, R. Michael Rich ${ }^{5}$, Suk-Jin Yoon ${ }^{2}$, \\ Sukyoung K. Yi ${ }^{2}$, Luciana Bianch ${ }^{6}$, Yongbeom Kang ${ }^{1}, \mathrm{KyeOngsook} \mathrm{LeE}^{1}$, Chul Chung ${ }^{2}, \mathrm{SANG}$ Yoon LeE $^{2}$, \\ Tom A. Barlow ${ }^{3}$, Karl Foster ${ }^{3}$, Peter G. Friedman ${ }^{3}$, D. Christopher Martin ${ }^{3}$, Patrick Morrissey $^{3}$, Susan G. NefF ${ }^{7}$, \\ David Schiminovich ${ }^{8}$, Mark Seibert $^{9}$, Ted K. Wyder ${ }^{3}$, Jose Donas $^{10}$, Timothy M. Heckman ${ }^{6}$, Barry F. Madore $^{9}$, \\ Bruno Milliard $^{10}$, Alex S. Szalay ${ }^{6}$, and Barry Y. Welsh ${ }^{11}$ \\ ${ }^{1}$ Department of Astronomy and Space Science, Chungnam National University, Daejeon 305-764, Republic of Korea \\ ${ }^{2}$ Center for Space Astrophysics, Yonsei University, Seoul 120-749, Republic of Korea \\ ${ }^{3}$ California Institute of Technology, MC 405-47, 1200 East California Boulevard, Pasadena, CA 91125, USA \\ ${ }^{4}$ Instituto de Astrofisica de Canarias, Via Lactea, E-38200 La Laguna, Tenerife, Spain \\ ${ }^{5}$ Department of Physics and Astronomy, University of California, Los Angeles, CA 90095, USA \\ ${ }^{6}$ Department of Physics and Astronomy, The Johns Hopkins University, Homewood Campus, Baltimore, MD 21218, USA \\ ${ }^{7}$ Laboratory for Astronomy and Solar Physics, NASA Goddard Space Flight Center, Greenbelt, MD 20771, USA \\ ${ }^{8}$ Department of Astronomy, Columbia University, New York, NY 10027, USA \\ ${ }^{9}$ Observatories of the Carnegie Institution of Washington, 813 Santa Barbara Street, Pasadena, CA 91101, USA \\ ${ }^{10}$ Laboratoire d'Astrophysique de Marseille, BP 8, Traverse du Siphon, 13376 Marseille Cedex 12, France \\ ${ }^{11}$ Space Sciences Laboratory, University of California at Berkeley, 601 Campbell Hall, Berkeley, CA 94720, USA \\ Received 2009 May 12; accepted 2009 June 12; published 2009 June 29
}

\begin{abstract}
We explore the age distribution of the globular cluster (GC) system of the nearby elliptical galaxy NGC 5128 using ultraviolet (UV) photometry from GALEX observations, with UV-optical colors used as the age indicator. Most GCs in NGC 5128 follow the general trends of GCs in M31 and the Milky Way in the UV-optical colorcolor diagram, which indicates that the majority of GCs in NGC 5128 are old similar to the age range of old GCs in M31 and the Milky Way. A large fraction of spectroscopically identified intermediate-age GC (IAGC) candidates with 3-8 Gyr are not detected in the far-UV (FUV) passband. Considering the nature of intermediateage populations being faint in the FUV passband, we suggest that many of the spectroscopically identified IAGCs may be truly intermediate in age. This is in contrast to the case of M31 where a large fraction of spectroscopically suggested IAGCs are detected in FUV and therefore may not be genuine IAGCs but rather older GCs with developed blue horizontal branch stars. Our UV photometry strengthens the results previously suggesting the presence of GC and stellar subpopulation with intermediate age in NGC 5128. The existence of IAGCs strongly indicates the occurrence of at least one more major star formation episode after a starburst at high redshift.
\end{abstract}

Key words: galaxies: individual (NGC 5128) - galaxies: star clusters - globular clusters: general - ultraviolet: galaxies

\section{INTRODUCTION}

Globular cluster (GC) systems provide the signatures of formation and assembly histories of their host galaxies assuming that major star formations in galaxies are accompanied with global GC formation. Several scenarios have been proposed to account for the observational properties obtained for the GC systems (see a comprehensive review of Brodie \& Strader 2006). Many aspects of those scenarios are in favor of the currently accepted hierarchical galaxy formation theory (Press \& Schechter 1974) rather than the monolithic formation at high redshift (Eggen et al. 1962; Larson 1974). In this galaxy formation paradigm, a constituent of galaxy mass including GCs is predicted to form through quiescent as well as merger/ interaction-driven star formation (Kaviraj et al. 2007b).

One of the best templates in the local universe for testing this scenario is the elliptical galaxy NGC 5128 due to its proximity. There have been several pieces of evidence supporting the picture that the NGC 5128 is the prototype for a postmerger elliptical galaxy (see Israel 1998 and references therein). Previous photometric and spectroscopic observations of GCs also suggest that merging and/or interaction events have played an important role in shaping its star cluster system (Peng et al. 2004a, 2004b; Woodley et al. 2007; Beasley et al. 2008).
Constraining the formation scenario of the NGC 5128 GC system requires the understanding of its global age distribution. Clusters younger than the bulk of their ancient Galactic counterparts are of particular interest because these objects represent the later stages of star formation histories in galaxies. Recent spectroscopic observations suggest that NGC 5128 hosts a cluster population significantly younger than the old GCs in the Milky Way and M31 (Peng et al. 2004b). Based on the spectroscopic observations for an increased sample of GCs, Beasley et al. (2008) reported the discovery of metal-rich, intermediateage GCs (IAGCs) with ages of 3-8 Gyr in NGC 5128. They propose that this population may be the by-product formed during merging events and/or interactions involving star formation and GC formation several gigayears ago.

However, it is important to note that age-dating of GCs via integrated spectra is hampered by the degeneracy between age and the existence of hot old stellar population (e.g., blue horizontal branch (HB) stars) affecting the strength of agesensitive line indices (Lee et al. 2000; Maraston et al. 2003; Thomas et al. 2003; Schiavon et al. 2004; Lee \& Worthey 2005; Trager et al. 2005; Cenarro et al. 2007). The effect of old blue HB stars in the integrated spectra can mimic young ages for old GCs, raising a cause of concern that may cast doubt on the intermediate-age nature of the GC in some galaxies. 
The UV colors (e.g., FUV $-V$ and FUV - NUV), on the other hand, are known to provide robust age estimation of simple stellar populations (e.g., Yi 2003; Rey et al. 2005, 2007; Kaviraj et al. 2007a; Bianchi et al. 2007). Kaviraj et al. (2007a) found that the age constraint is far superior when UV photometry is added to the optical colors and its quality is comparable or marginally better than the case of utilizing spectroscopic indices.

With the new approach using UV observations, in this Letter, we take advantage of the combination of available optical photometry and GALEX UV photometry to confirm the existence of IAGCs and to explore the age distribution of the NGC 5128 GC system. In the following sections, we emphasize the importance of the UV photometry as a probe of IAGCs in general. Comparing these with GCs in M31 and the Milky Way with the aid of our population models, we describe the overall age distribution of GCs and identification of IAGCs in NGC 5128. In this Letter, we denote IAGCs as those having ages $\sim 3-8$ Gyr.

\section{OBSERVATIONS AND DATA ANALYSIS}

GALEX (Martin et al. 2005) imaged one 1.25 degree circular field centered on 26 arcmin east and 7 arcmin north of the NGC 5128 core in two UV bands: FUV (1350-1750 $\AA$ ) and NUV (1750-2750 A). The images were obtained on 2004 April, and are included in the GALEX fourth and fifth data release (GR4/ GR5). ${ }^{12}$ Total integration times were $30,428 \mathrm{~s}$ and $20,072 \mathrm{~s}$ for NUV and FUV, respectively. Preprocessing and calibrations were performed via the GALEX pipeline (Morrissey et al. 2005, 2007). GALEX image has a sampling of $1.5 \operatorname{arcsec}$ pixel $^{-1}$ which corresponds to $19 \mathrm{pc}$ at the distance of NGC 5128 (3.9 Mpc; Woodley et al. 2007).

Using the DAOPHOTII/ALLSTAR package (Stetson 1987), we performed aperture photometry for all detected point sources in the GALEX NGC 5128 field. Aperture corrections were derived using moderately bright, isolated objects. Flux calibrations were applied to bring all measurements into the $\mathrm{AB}$ magnitude system (Oke 1990; Morrissey et al. 2005, 2007).

Point sources in our GALEX photometry were cross-matched using a matching radius of 3 arcsec with the catalog of Woodley et al. (2007). This catalog provides positions as well as optical magnitudes and mean radial velocities for $415 \mathrm{GCs}$ in NGC 5128. All spurious and ambiguous sources were rejected based on visual inspection. The final sample of visually confirmed GCs are 157 and 35 in NUV and FUV, respectively. We adopted a foreground reddening value of $E(B-V)=0.11$ for NGC 5128 (Woodley et al. 2007) and use the reddening law of Cardelli et al. (1989). The full UV catalog and discussion of the UV properties of GCs in NGC 5128 will be presented in a forthcoming paper. Figure 1 shows the optical color-magnitude diagram (CMD) of GCs in NGC 5128 detected in the NUV and FUV bandpasses. For comparison, we overplot GCs in M31 detected from GALEX observations (Rey et al. 2007). The CMD shows that most of the UV-detected objects in NGC 5128 and M31 have similar distribution and are confined to $V-I<1.05$.

\section{ULTRAVIOLET AS A PROBE OF INTERMEDIATE-AGE GLOBULAR CLUSTERS}

FUV flux plays an important role in identifying IAGCs. Young $(<1 \mathrm{Gyr})$ stellar populations emit a substantial portion of their flux in the UV. Metal-poor old (> $10 \mathrm{Gyr}$ ) stellar

\footnotetext{
12 http://galex.stsci.edu/gr4
}

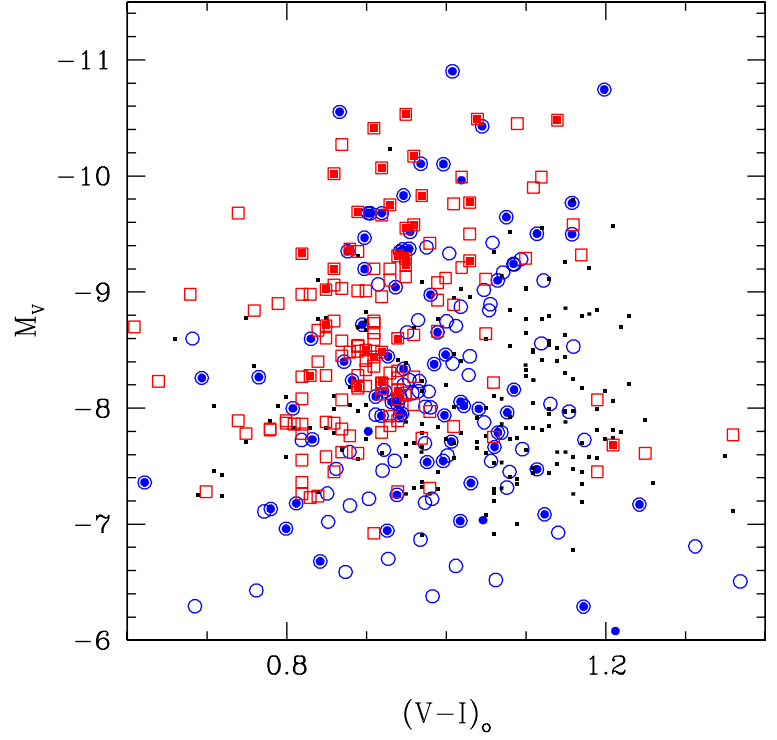

Figure 1. $M_{V}$ vs. $(V-I)_{o}$ CMD of GALEX UV-detected GCs in NGC 5128 (squares) and M31 (circles; Rey et al. 2007). Open and filled symbols are objects detected in NUV and FUV, respectively. We note that all FUV-detected GCs in NGC 5128 are detected in NUV. The small dots indicate GCs in NGC 5128 that are not detected in GALEX UV observations.

populations also show large FUV to optical flux ratio due to the contribution of hot HB stars. On the contrary, intermediateage ( $\sim 3-8 \mathrm{Gyr})$ populations emit negligible amount of FUV flux since the constituent stars are not hot enough to produce a significantly large FUV flux (see Figure 1 of Kaviraj et al. 2007a). Consequently, if the IAGC candidates identified by spectroscopic observations are truly intermediate in age, they should be very faint or not detected in our GALEX FUV photometry given our integration time and the detection limit (Lee \& Worthey 2005; Rey et al. 2007; Kaviraj et al. 2007a).

The first use of UV color as a tool for identifying IAGCs was demonstrated in our M31 study (see Rey et al. 2007). Spectroscopic observations of M31 clusters have suggested the existence of IAGCs with a mean age of $\sim 5 \mathrm{Gyr}$ (Burstein et al. 2004; Beasley et al. 2005; Puzia et al. 2005). However, based on GALEX FUV detections of more than half of M31 IAGC candidates, Rey et al. (2007) suggested that a large fraction of the spectroscopically identified IAGCs may not be truly intermediate in age but are rather old GCs with a developed blue HB sequence. Among the 42 GCs in M31 whose ages are estimated by Kaviraj et al. (2007a), we find that four IAGC candidates turn out to be old GCs with $>12$ Gyr. By comparing the mass-to-light ratios of three IAGC candidates in M31 with those of old GCs, Strader et al. (2009) also found no evidence that M31 IAGC candidates are of intermediate in age.

The most direct way to identify genuine IAGCs is to inspect CMDs of the clusters of interest. In the case of M31, Hubble Space Telescope (HST) CMDs of two IAGC candidates B311 and B058 exhibit clearly developed blue HB sequences (Rich et al. 2005). In a separate study, Chandar et al. (2006) showed that a star cluster in M33, C38, is a genuine IAGC with age $\sim 2$ 5 Gyr based on the HST CMD and Balmer line measurements. It is important to note that this cluster is also confirmed to be a genuine IAGC using the GALEX FUV observations of M33 (S. T. Sohn et al. 2009, in preparation). In any case, UV-optical color can be used to discriminate genuine IAGCs from the old GCs masquerading as IAGCs. 


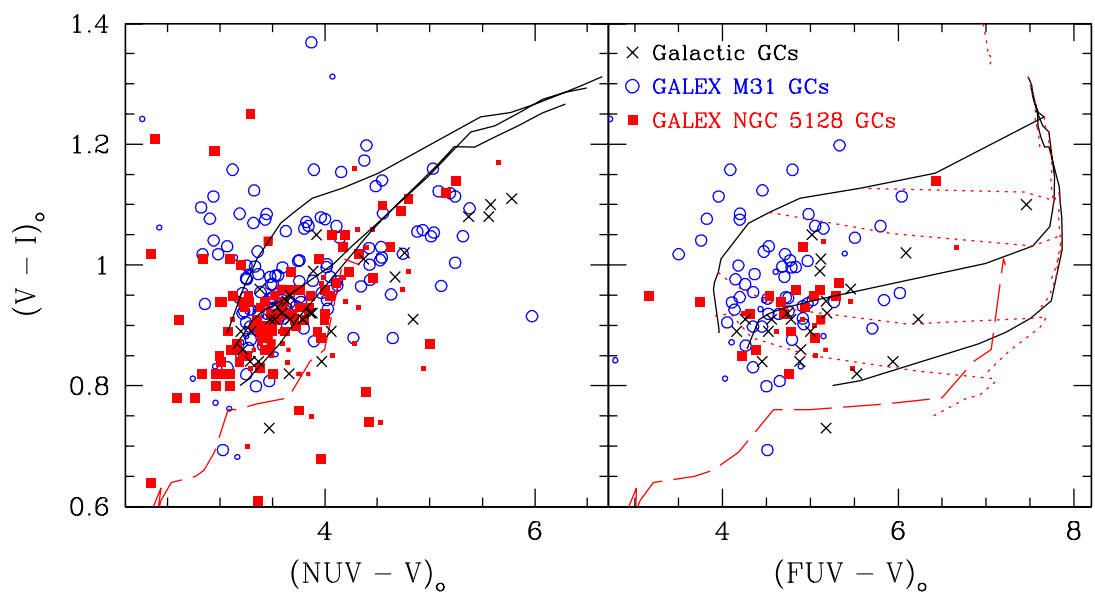

Figure 2. $(V-I)_{o}$ vs. (UV $\left.-V\right)_{o}$ diagrams of NGC 5128 (filled squares), the Milky Way (crosses), and M31 (open circles) GCs. Large and small squares indicate NGC $5128 \mathrm{GCs}$ with small and large magnitude errors in the UV passband ( $0.2 \mathrm{mag}$ for NUV and $0.3 \mathrm{mag}$ for FUV as the border line), respectively. Large circles are M31 GCs with $E(B-V)<0.16$ from Barmby et al. (2000). Small circles are M31 GCs with no available reddening information in Barmby et al., assuming that they are only affected by the Galactic foreground reddening of $E(B-V)=0.10$. We superpose our simple stellar population model lines with old (10, 12 , and 14 Gyr; solid lines from bottom to top) and young (long dashed line for $1 \mathrm{Gyr}$ ) ages. The dotted lines represent isometallicity lines varying from $[\mathrm{Fe} / \mathrm{H}]=-2.0$ to +0.5 dex (from bottom to top). There is no significant difference of distribution between red $\left[(V-I)_{o}>0.8\right]$ and old GCs in the three galaxies.

\section{AGE DISTRIBUTION OF GLOBULAR CLUSTERS IN NGC 5128}

\subsection{Old Globular Clusters}

Figure 2 shows the $V-I$ versus $U V-V$ diagrams. We compare our NGC 5128 sample with those of the Milky Way (crosses; Sohn et al. 2006) and M31 (open circles; Rey et al. 2007) GCs whose age distributions are reasonably well constrained. We also show our simple stellar population (SSP) models constructed using the Yonsei Evolutionary Population Synthesis (YEPS) code (Lee et al. 2000, 2005; Rey et al. 2005, 2007; Yoon et al. 2006, 2008).

In Figure 2, NGC 5128 GCs appear to show tight distribution around the $12 \mathrm{Gyr}$ model line similar to that of the Milky Way, while GCs in M31 are rather scattered in $V-I$. This is partly due to the detection limit of optically red GCs in NGC 5128 (see Figure 1) and an insufficient sample of Milky Way GCs obtained from previous UV observations of various satellites (see Sohn et al. 2006). Furthermore, Rey et al. (2007) reported the existence of UV-bright metal-rich GCs with extreme hot blue HB stars in M31 (e.g., NGC 6388 and NGC 6441 in the Milky Way; Rich et al. 1997). In this regard, some of the red $(V-I>1.0)$ M31 GCs that show UV excess with respect to the 14 Gyr model line may be such peculiar objects. Considering these points, at a fixed $V-I$, the majority of GCs in three galaxies show similar spread in the UV $-V$ colors and are well accounted for by the 10-14 Gyr model lines. This suggests that the mean age and age spread, at least, for old ( $\geqslant 10 \mathrm{Gyr}$ ) GCs are similar among GC systems of different galaxies (the Milky Way, M31, and NGC 5128).

\subsection{Intermediate-Age Globular Clusters}

Beasley et al. (2008) found a population of intermediate-age and predominantly metal-rich $([\mathrm{Z} / \mathrm{H}]>-1.0) \mathrm{GCs}(15 \%$ of the sample) from their spectroscopic observations. Among the 21 IAGC candidates (age 3-8 Gyr) identified by Beasley et al. (2008), we detect only two in the GALEX FUV passband.

In Figure 3, we show the $V-I$ versus FUV $-V$ diagram for the spectroscopically identified IAGC candidates in NGC 5128 (filled squares) and M31 (filled circles) detected in the

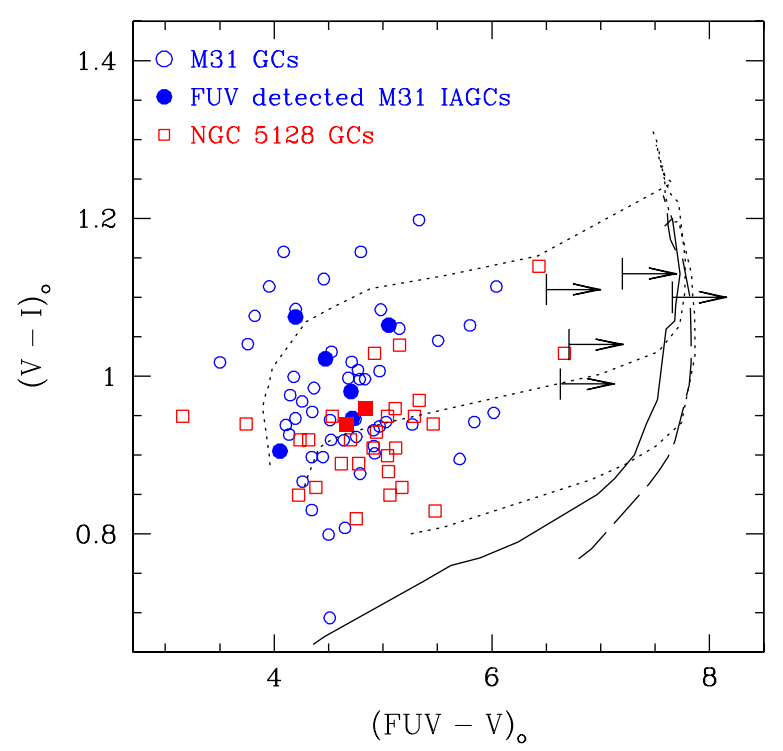

Figure 3. $(V-I)_{o}$ vs. (FUV $\left.-V\right)_{o}$ color-color diagram for the spectroscopically classified IAGC candidates in NGC 5128 (filled squares) and M31 (filled circles) detected in the GALEX FUV passband. The model lines for intermediate ages (solid line for $3 \mathrm{Gyr}$ and long dashed line for $8 \mathrm{Gyr}$ ) are overplotted in addition to the old $(10,12$, and $14 \mathrm{Gyr}$; dotted lines from bottom to top) ones. All IAGC candidates of NGC 5128 and M31 detected in the FUV show similar distribution to that of old GCs (open circles and squares) with $>10$ Gyr. The color limit for each IAGC candidate of NGC 5128 not detected in the FUV is plotted with a vertical bar and a horizontal arrow pointing to the redder color. Color limits were determined by adopting the flux of the faintest FUV-detected GC in NGC 5128.

GALEX FUV passband. Population model lines covering range of intermediate ( 3 and $8 \mathrm{Gyr}$ ) and old (10,12, and $14 \mathrm{Gyr}$ ) ages are overplotted for guidance. It is immediately apparent that all of the IAGC candidates of NGC 5128 and M31 detected in the FUV show similar distribution to those of old GCs with $>10$ Gyr, i.e., all FUV-detected IAGC candidates have significantly bluer FUV $-V$ colors than the 3 and 8 Gyr model lines. This indicates that IAGC candidates detected in the FUV are in fact old GCs ( $\geqslant 10 \mathrm{Gyr})$ containing developed blue HB populations that contribute to the strong Balmer absorption lines. 
It is important to note that, as shown in Figure 3, most M31 IAGC candidates with $E(B-V)<0.16$ are detected in the GALEX FUV (6 out of 7; see Rey et al. 2007 for the details). If we restrict the sample of M31 IAGC candidates to match the observed optical brightness and color range $\left(M_{V}<-8\right.$ and $V-I<1.05$; see Figure 1) of the FUV-detected sample of NGC $5128 \mathrm{GCs}$, four out of the five M31 IAGC candidates are detected in the FUV. In the case of NGC $5128 \mathrm{GCs}$, only two out of the nine IAGC candidates are detected in the FUV. Since all of the NGC 5128 GCs detected in the FUV cover a similar range of $(F U V-V)_{o}$ colors of FUV-detected IAGC candidates in M31, most, if not all, spectroscopically identified IAGC candidates in NGC 5128 are not likely to be as bright as those in M31.

Among the 21 IAGC candidates identified by Beasley et al. (2008), 12 GCs are detected in the GALEX NUV but not in the FUV. Whereas the FUV flux of old ( $>8 \mathrm{Gyr}$ ) GC is almost entirely dominated by stars in the hot HB sequence, the NUV flux is influenced by both the HB stars and those on the mainsequence turnoff. In this regard, we cannot rule out that some of the NUV-detected IAGC candidates are truly intermediate in age, despite the fact that NUV $-V$ is relatively insensitive to age variations compared to the FUV $-V$ (see Figure 2). To test this hypothesis, in Figure 3, we show the bluer limits of the NUV-detected IAGC candidates having similar $V$ magnitudes of FUV-detected IAGCs. Most of the color limits are consistent with the NUV-detected IAGC candidates being 3-8 Gyr in age. In summary, our UV photometry suggests that NGC 5128 does possess a non-negligible fraction of IAGCs that are intrinsically faint in the FUV as proposed by previous spectroscopic studies.

\section{DISCUSSION AND CONCLUSIONS}

In this work, we explored the age distribution of GCs in the giant elliptical galaxy NGC 5128 using the UV colors. The majority of NGC 5128 GCs show age ranges similar to old GCs in M31 and the Galactic halo. Our most important result is that a large fraction of IAGCs identified by the spectroscopic observations are not detected in the GALEX FUV passband and therefore may be truly intermediate in age. This is in contrast to the case of M31 GCs where the majority of IAGC candidates turned out to be old GCs with developed HB sequence based on their FUV $-V$ colors (see Rey et al. 2007).

The existence of IAGCs in NGC 5128 supports the galaxy formation scenario accompanied with at least two major star formation episodes, e.g., hierarchical assembly of the protogalactic fragments or disks (Bekki et al. 2003; Beasley et al. 2002, 2003; Yi et al. 2004; Kaviraj et al. 2005). In these models, some of the metal-rich GCs are formed from pre-enriched gas clouds and are on average younger than the metal-poor GCs. Based on the kinematic analysis in combination with the age distribution of GCs, an alternative mechanism may have taken place where the NGC 5128 formed its main body at early times and has gradually built up by minor mergers and gas-rich satellite accretions accompanied by star formation episodes (Woodley 2006; Woodley et al. 2007).

The presence of IAGCs in NGC 5128 has an interesting implication for the recent star formation (RSF) recently discovered using the large GALEX UV sample of early-type galaxies at different redshifts $(0<z<1$; e.g., Yi et al. 2005; Kaviraj et al. 2007b, 2008; Schawinski et al. 2007). Kaviraj et al. (2008) found that high-redshift early-type galaxies in the range of $0.5<z<1$ exhibit typical RSFs in addition to the case of low-redshift $(0<z<0.1)$ early-type galaxies. This provides a compelling evidence that RSFs in early-type galaxies are non-negligible over the last 8 billion years. Furthermore, Kaviraj et al. (2008) suggest that up to $10 \%-15 \%$ of the mass of luminous $\left(-23<M_{V}<-20.5\right)$ early-type galaxies such as NGC $5128\left(M_{V}=-21.08\right.$; Gil de Paz et al. 2007) may have formed after $z=1$. These results imply that early-type galaxies in the local universe are likely to possess intermediate-age stellar populations. In this respect, IAGCs in NGC 5128 may be considered as relics of residual star formations that occurred during the last few billion years.

UV observations of the GC systems have been shown to provide important insights into the identification of IAGCs which is at present difficult to be identified solely by spectroscopic observations. In particular, the Balmer line strengths themselves cannot reliably pin down the age of GCs because of the degeneracy between age and HB morphology. FUV colors, on the other hand, can verify the contribution from hot stellar populations in GCs and help identify the true IAGCs. Deep UV observations are highly anticipated for other galaxies with IAGC candidates identified by various spectroscopic and near-infrared photometric observations.

We thank Sugata Kaviraj for useful suggestions on the manuscript. This work was supported by the Korea Research Foundation Grant funded by the Korean Government (MOEHRD; KRF-2005-202-C00158) and the Korea Science and Engineering Foundation (KOSEF) through the Astrophysical Research Center for the Structure and Evolution of the Cosmos (ARCSEC). GALEX (Galaxy Evolution Explorer) is a NASA Small Explorer, launched in 2003 April. We gratefully acknowledge NASA's support for construction, operation, and science analysis for the GALEX mission, developed in cooperation with the Centre National d'Etudes Spatiales of France and the Korean Ministry of Science and Technology.

Facilities: GALEX

\section{REFERENCES}

Barmby, P., Huchra, J. P., Brodie, J. P., Forbes, D. A., Schroder, L. L., \& Grillmair, C. J. 2000, AJ, 119, 727

Beasley, M. A., Baugh, C. M., Forbes, D. A., Sharples, R. M., \& Frenk, C. S. 2002, MNRAS, 333, 383

Beasley, M. A., Bridges, T., Peng, E., Harris, W. E., Harris, G. L. H., Forbes, D. A., \& Mackie, G. 2008, MNRAS, 386, 1443

Beasley, M. A., Harris, W. E., Harris, G. L. H., \& Forbes, D. A. 2003, MNRAS, 340,341

Beasley, M. A., et al. 2005, AJ, 129, 1412

Bekki, K., Harris, W. E., \& Harris, G. L. H. 2003, MNRAS, 338, 587

Bianchi, L., et al. 2007, ApJS, 173, 659

Brodie, J. P., \& Strader, J. 2006, ARA\&A, 44, 193

Burstein, D., et al. 2004, ApJ, 614, 158

Cardelli, J. A., Clayton, G. C., \& Mathis, J. S. 1989, ApJ, 345, 245

Cenarro, A. J., Beasley, M. A., Strader, J., Brodie, J. P., \& Forbes, D. A. 2007, AJ, 134,391

Chandar, R., Puzia, T. H., Sarajedini, A., \& Goudfrooij, P. 2006, ApJ, 646, L107 Eggen, O. J., Lynden-Bell, D., \& Sandage, A. R. 1962, ApJ, 136, 748

Gil de Paz, A., et al. 2007, ApJS, 173, 185

Israel, F. P. 1998, A\&AR, 8, 237

Kaviraj, S., Ferreras, I., Yoon, S.-J., \& Yi, S. K. 2005, A\&A, 439, 913

Kaviraj, S., Rey, S.-C., Rich, R. M., Yoon, S.-J., \& Yi, S. K. 2007a, MNRAS, 381,74

Kaviraj, S., et al. 2007b, ApJS, 173, 619

Kaviraj, S., et al. 2008, MNRAS, 388, 67

Larson, R. B. 1974, MNRAS, 166, 585

Lee, H.-c., \& Worthey, G. 2005, ApJS, 160, 176

Lee, H.-c., Yoon, S.-J., \& Lee, Y.-W. 2000, AJ, 120, 998

Lee, Y.-W., et al. 2005, ApJ, 621, 57L

Maraston, C., Greggio, L., Renzini, A., Ortolani, S., Saglia, R. P., Puzia, T. H., \& Kissler-Patig, M. 2003, A\&A, 400, 823 
Martin, D. C., et al. 2005, ApJ, 619, L1

Morrissey, P., et al. 2005, ApJ, 619, L7

Morrissey, P., et al. 2007, ApJS, 173, 682

Oke, J. B. 1990, AJ, 99, 1621

Peng, E. W., Ford, H. C., \& Freeman, K. C. 2004a, ApJS, 150, 367

Peng, E. W., Ford, H. C., \& Freeman, K. C. 2004b, ApJ, 602, 705

Press, W. H., \& Schechter, P. 1974, ApJ, 187, 425

Puzia, T. H., Perrett, K. M., \& Bridges, T. J. 2005, A\&A, 434, 909

Rey, S.-C., et al. 2005, ApJ, 619, L119

Rey, S.-C., et al. 2007, ApJS, 173, 643

Rich, R. M., et al. 1997, ApJ, 484, L25

Rich, R. M., et al. 2005, AJ, 129, 2670

Schawinski, K., et al. 2007, ApJS, 173, 512

Schiavon, R. P., Rose, J. A., Courteau, S., \& MacArthur, L. A. 2004, ApJ, 608, 33

Sohn, S. T., et al. 2006, AJ, 131, 866
Stetson, P. B. 1987, PASP, 99, 191

Strader, J., Smith, G. H., Larsen, S., Brodie, J. P., \& Huchra, J. P. 2009, AJ, 138, 547

Thomas, D., Maraston, C., \& Bender, R. 2003, MNRAS, 339, 897

Trager, S. C., Worthey, G., Faber, S. M., \& Dressler, A. 2005, MNRAS, 362,

Woodley, K. A. 2006, AJ, 132, 2424

Woodley, K. A., Harris, W. E., Beasley, M. A., Peng, E. W., Bridges, T. I., Forbes, D. A., \& Harris, G. L. H. 2007, AJ, 134, 494

Yi, S. K. 2003, ApJ, 582, 202

Yi, S. K., Peng, E., Ford, H., Kaviraj, S., \& Yoon, S.-J. 2004, MNRAS, 349, 1493

Yi, S. K., et al. 2005, ApJ, 619, L111

Yoon, S.-J., Joo, S.-J., Ree, C. H., Han, S. I., Kim, D.-G, \& Lee, Y.-W. 2008, ApJ, 677,1080

Yoon, S.-J., Yi, S. K., \& Lee, Y.-W. 2006, Science, 311, 1129 